\title{
Histological analysis of post-eruption tooth wear adaptations, and ontogenetic changes in tooth implantation in the acrodontan squamate Pogona vitticeps
}

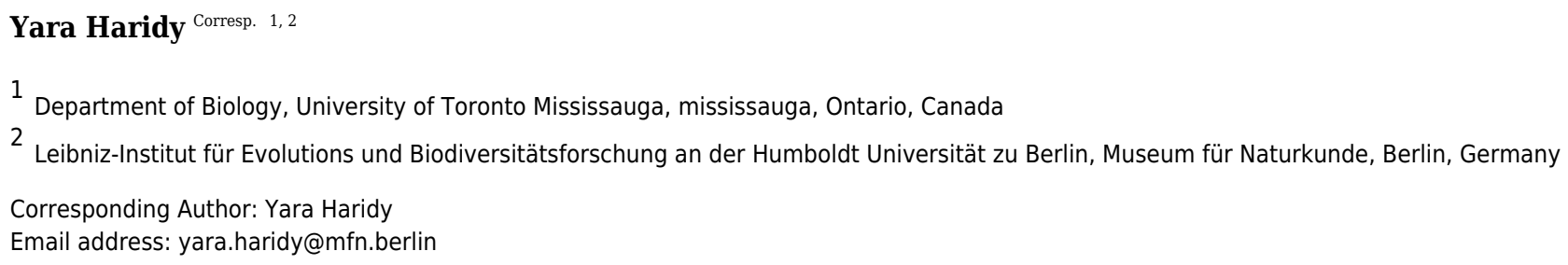

Teeth have been a focus of research in both extinct and extant taxa alike; a significant portion of dental literature is concerned with dental patterning and replacement. Most non-mammalian vertebrates continuously replace their dentition but an anomalous group of squamates has forgone this process in only having one tooth generation; these squamates all have apically implanted teeth, a condition known as acrodonty. Acrodont dentition and various characteristics attributed to it, including a lack of replacement, have often been defined ambiguously. This study explores this type of implantation through histology in the ontogeny of the acrodont agamid Pogona vitticeps. The non-replacing teeth of this squamate provides an opportunity to study wear adaptations, maintenance of occlusion in a non-mammalian system, and most importantly post-eruption changes in the tooth bone interface. In this study the post-eruption changes combined with dental wear likely gives the appearance of acrodont implantation. 
1 Histological analysis of post-eruption tooth wear adaptations, and ontogenetic changes in 2 tooth implantation in the acrodontan squamate Pogona vitticeps

3 Yara Haridy ${ }^{1,2}$

$4{ }^{1}$ Department of Biology, University of Toronto Mississauga, 3359 Mississauga Road,

5 Mississauga, Ontario, L5L 1C6, Canada

$6 \quad{ }^{2}$ Museum für Naturkunde, Leibniz-Institut für Evolutions und Biodiversitätsforschung an der

7 Humboldt Universität zu Berlin, Invalidenstrasse 43, 10115 Berlin, Germany

8

9 Corresponding Author:

10 Yara Haridy ${ }^{1}$

11 Email address: yara.haridy@mfn.berlin

12

13

14

15

16

17

18

19

20

21 
22 Abstract. Teeth have been a focus of research in both extinct and extant taxa alike; a significant 23 portion of dental literature is concerned with dental patterning and replacement. Most non-

24 25 26 mammalian vertebrates continuously replace their dentition but an anomalous group of squamates has forgone this process in only having one tooth generation; these squamates all have apically implanted teeth, a condition known as acrodonty. Acrodont dentition and various characteristics attributed to it, including a lack of replacement, have often been defined ambiguously. This study explores this type of implantation through histology in the ontogeny of the acrodont agamid Pogona vitticeps. The non-replacing teeth of this squamate provides an opportunity to study wear adaptations, maintenance of occlusion in a non-mammalian system, and most importantly post-eruption changes in the tooth bone interface. In this study the posteruption changes combined with dental wear likely gives the appearance of acrodont implantation.

\section{Introduction}

Reptilian dentition has been extensively studied in both extinct and extant taxa, and for the vast majority of these taxa, there is constant replacement of teeth, a condition known as polyphyodonty. However, in a subset of reptiles, there has been an evolutionary cessation of replacement, a condition known as monophyodonty. Among lepidosaurian reptiles, this suppression of replacement is limited to Sphenodontidae (Rhynchocephalia), Chamaeleonidae, and Agamidae, with the latter two being grouped within the clade Acrodonta (Squamata) (Pyron et al., 2013). This squamate group is aptly named for the acrodont style implantation of the dentition, and all acrodontians have apically implanted teeth making up the majority of their dentulous surface (Edmund, 1960; Peyer,1968; Zaher and Reippel, 1999; Cooper et al.,1970 Jenkins et al., 2017). This is in contrast to the condition found in most squamates and other reptiles (e.g., Zaher and Rippel, 1999; Delgado et al., 2003a; LeBlanc and Reisz, 2015) in which the tooth is implanted to the lingual surface of the jaw bone, a condition known as pleurodonty that is exemplified in taxa like Iguana iguana (Throckmorton, 1976; Montanucci, 2008; Kline and Cullum, 2017). The most studied form of implantation among tetrapods is thecodonty, where the tooth is implanted in a deep socket; this form of implantation is found in all mammals and also occurs within crocodilians and in many extinct archosaurs (e.g., Zaher and Reippel, 
52 1999;Brown et al., 2015; García and Zurriaguz, 2016). Tooth implantation should not be

53 conflated with tooth attachment, which refers to the tissue that attaches the tooth to the dentulous

54 bone. This study is primarily concerned with implantation, and how ontogenetic change can

55 influence the appearance of implantation categories.

True teeth in most non-mammalian vertebrates are well known for their extensive replacement patterns through their life (Zaher and Rieppel, 1999; Delgado et al., 2003b; LeBlanc and Reisz, 2015; LeBlanc et al., 2016b); therefore any condition that deviates from polyphyodonty is unusual and worthy of study. An important example of non-polyphyodont dentition is seen in mammals, in which there are only two generations of dentition, the deciduous teeth and the permanent teeth (Sire et al., 2002). Some mammals such as shrews have even forgone the deciduous phase by resorbing the dentition prior to eruption, functionally giving them one tooth generation (Järvinen et al., 2009). Acrodonty in Reptilia is largely associated with a lack of tooth replacement (e.g., Zaher and Rippel, 1999; Smirina and Ananjeva, 2007), an association that often alludes to causality but that is never stated outright. At first glance it seems understandable that acrodont implantation and monophyodonty be associated this is because most acrodont squamates have both acrodont and pleurodont dentition and in individuals with both pleurodont and acrodont dentitions, the pleurodont teeth are replaced, yet the acrodont teeth are not (Cooper et al. 1970). This discrepancy seems to have cemented the idea that acrodonty somehow inherently disrupts or inhibits replacement. While extant acrodont squamates are monophyodont, it is important to note that acrodonty is also found in some extinct reptiles 72 (Simões et al., 2015; Haridy et al., 2018) and in non-reptilian vertebrates such as piranhas 73 (Shellis and Berkovitz, 1976), all of which replace their dentition.

The supposed function of constant replacement is to avoid excessive wear (Throckmorton, 1979; Benton, 1984; Erickson, 1996); accordingly, higher rates of replacement are often seen in herbivorous taxa, as their fibrous diet and frequency of mastication requires a constant renewal of their dentition. In extreme instances, there are examples of starvation in herbivorous mammals that lack continuous replacement and that have worn their dentition to such a degree such that it is no longer functional (Spencer, 2005). Therefore the lack of replacement in acrodont squamates raises the question: if acrodontians that lack tooth replacement are relatively long-lived (Zari, 1999; Smirina and Ananjeva, 2017), how are they 
82 able to maintain a viable occlusal surface during later stages of life? More specifically: (1) how

83 do acrodontians adapt to dental wear at a tissue level, and (2) how do the mandibular and

84 maxillary teeth stay in occlusion through ontogeny without replacement or a ligamentous

85 attachment? The former of the two questions has been partially addressed through histology of

86 the acrodontians Uromastyx aegyptia (Throckmorton, 1979) and Chamaeleo calyptratus

87 (Buchtová et al., 2013; Dosedělová et al., 2016a), and members of acrodontan Uromastycinae

88 and Chamaeleoninae (Pyron et al., 2013). These studies found that these acrodontians have

89 peculiar way to combat wear - they secondarily infill the teeth with bone and/or dentine and

90 remodel the underlying jaw bone into more compact bone. There are six other groups within

91 Acrodonta that presumably also lack replacement and have undocumented wear adaptations

92 (Pyron et al., 2013). This study tests if the agamid Pogona vitticeps (the central bearded dragon)

93 conforms at a tissue level to the wear adaptation patterns of other acrodontians previously

94 documented in literature.

To better understand the relationship between acrodonty, monophyodonty, and wear adaptations, the implantation mode itself should be more thoroughly examined, particularly when it comes to acrodonty it is the least studied form of implantation. Acrodont implantation has been ambiguously defined at best, with various authors describing the mode of implantation as: (1) apically placed teeth (Edmund, 1960), (2) teeth ankylosed to the margin of the jaw (Motani, 1997), (3) teeth fused to the edge of the jaw bone via undefined tissues (Peyer,1968). Most definitions used acrodontian squamates or rhynchocephalians as representative groups for acrodont implantation, with acrodonty used as a phylogenetic character (e.g., Zaher and Rieppel, 1999). In this paper we use the most general definition of acrodonty as established by Edmund (1960), referring only to the apical position of the teeth in relation to the jaw.

The classic categories of acrodonty, pleurodonty, and thecodonty have been used as descriptors, as well as phylogenetic characters (Zaher and Rippel, 1999), and continue in even the latest literature (Jenkins et al., 2017) but have been called into question by Estes et al. (1988) who suggested these categories are artificial and are not likely representative of natural groupings. To ground truth the use of acrodontians as acrodont representatives the natural question becomes: are the acrodont teeth of acrodontians really acrodont? It was proposed that ichthyosaurs and alligators change implantation types ontogenetically by growth in the jaw 
112 ramus and additional ossification on the lingual side (Motani, 1997), alligators go from

113 pleurodont to thecodont, and the ichthyosaurs become subthecodont from their juvenile state of

114 pleurodonty.This study explores the ontogenetic change in the teeth of Pogona vitticeps, with a

115 focus on comparisons of wear adaptations of this member of Agamidinae to those previously

116 described in Uromastycinae and Chamaeleoninae. This study aims to explain: (1) Do tissue level

117 wear adaptations documented in other acrodontians extend to P. vitticeps? (2) How do

118 monophyodont reptiles that lack a ligamentous attachment maintain occlusion through ontogeny?

119 (3) Are the teeth of these reptiles truly acrodont, and does acrodonty limit tooth replacement as

120 indicated by literature? This is the first study to document the change in implantation types

121 through ontogeny due to osteological remodeling of the dentary and the tooth body, with

122 evidence of a change from pleurodont implantation to acrodont implantation in a modern

123 squamate. This has implications on how we view implantation categories as they are likely to be

124 more ontogenetically variable than previously thought.

\section{Materials and Methods}

In this study, the genus Pogona was represented by the central bearded dragon $(P$.

127 vitticeps). Thirty-seven specimens (S. Figure1) of $P$. vitticeps from the Royal Ontario Museum

128 (ROM) recent osteology collection were externally examined, and all measurements were taken

129 prior to skeletonizing which allowed the species to be identified. Specimens sectioned here were

130 captive bred and donated to the ROM. All specimens were photographed using a Canon

131 EOS40D prior to sectioning. To access normally inaccessible anatomical features of the lower

132 jaw and its dentition, 5 specimens were sectioned in both coronal and longitudinal planes.

133 Pogona vitticeps was sectioned at two ontogenetic stages, a juvenile stage (ROM R8234, ROM

134 R8510), and an adult stage (ROM R8507, ROM R8189, ROM R9422). The illustrations and

135 diagrams found in the figures were made using Adobe Photoshop CS6 and Adobe Illustrator 136 CS6.

137 Histology

138 All thin sections were made following the ROM histology protocol and were executed in 139 the ROM vertebrate paleontology thin sectioning facility. Specimens were embedded in AP 140 Castolite acrylic resin, vacuumed, and left to cure for a minimum of 24 hours. All specimens 
141 were cut using a Buhler Isomet 1000 wafer saw at a low speed of $275 \mathrm{rpm}$. The specimens were

142 mounted on plexiglass slides using Scotch-Weld SF-100 cyanoacrylate. The slides were then

143 mounted on the Hillquist grinding cup and ground down using the grinding cup until optical

144 clarity was achieved; subsequently the specimen was manually ground using progressively finer

145 grit suspensions on glass plates, beginning with a 600-grit silicon carbide powder and working

146 down to a 1-micron aluminum oxide powder. All slides were imaged using a Nikon DS-Fi1

147 camera mounted to a Nikon AZ 100 microscope fitted with crossed-polarizing and lambda filters

148 and an oblique illumination slider and NIS-Elements software registered to R. R. Reisz of the

149 University of Toronto Mississauga.

\section{Results}

\section{External anatomy}

152

The external morphology of both the mandible and dentition is noticeably different

153 between the juvenile and adult specimens of Pogona vitticeps (Figs. 1A,B). Several specimens

154 that represented a relatively complete ontogenetic sequence were examined (see Table1 in

155 supplementary material); for the sake of brevity, two representative specimens, an adult (ROM

156 R8507) and a juvenile (ROM R8234), were chosen for external description. The juvenile

157 specimen (ROM R8234) has fewer tooth positions (16 tooth positions) on the dentary; however,

158 smaller individuals were found to have as few as 10 teeth. There are external mandibular features

159 of ROM R8234 that identify it as juvenile: (1) the symphysis is poorly ossified, (2) the mandible

160 is less robust in bone density, which is externally apparent as a character of bone opacity, and in

161 dorsoventral width, and (3) the mandible is relatively short, with the dentulous region making up

162 more than half the total length. The wear facets on the labial surface of the dentary are not as

163 well developed in the juvenile specimen as those seen in the adults. This specimen of $P$. vitticeps

164 is found to have two teeth implanted in a pleurodont fashion; these occupy the first two tooth

165 positions on the rostral end of the mandible (Fig. 1B). The pleurodont teeth are conical and taper

166 to a point; this represents the most common condition among many, but not all agamids (Cooper

167 et al. 1970). Posterior to the pleurodont dentition, the remaining tooth row has been described in

168 literature to be acrodont and is the focus of this study (Cooper et al., 1970). The acrodont teeth in

$169 P$. vitticeps are triangular and mediolaterally compressed and lack the multi-cuspid morphology 
170 that other acrodontians (S.Figure 1), like chameleons and Uromastyx display. Compared to the

171 older anterior teeth, the teeth are larger in size posteriorly correlating with age,with the posterior

172 teeth being the largest and the youngest. It appears that the newest tooth is not ankylosed to the

173 jaw bone prior to the development of the next tooth in the series. Instead, the newest tooth

174 appears be attached to the jaw only by soft tissue. Desiccated fibrous tissue separates it from the

175 bone in skeletonized material, (Fig. 2); this is readily recognizable by external observation,

176 because the last two teeth are not oriented at an angle that is congruent with the rest of the

177 dentition (Fig. 1A). This was also observed in several other juvenile specimens in which it is

178 observed only in the posteriormost teeth. Lastly, the newest and last tooth position is growing

179 directly against, and partially resorbing (Fig. 1A), the coronoid process, which does not allow

180 sufficient space for the next more posterior tooth position.

181

As with the juveniles, several adult individuals were examined, and a representative

182 specimen chosen. Several changes correlated with size and thus presumably with ontogeny can

183 be recognized in the adult specimen of Pogona vitticeps (ROM R8507) including an increase in

184 the number of tooth positions. The specimen chosen for sectioning has 17 tooth positions,

185 although other specimens have been found to have as many as 19 tooth positions. In most

186 specimens a definite tooth count was difficult to ascertain via examination of the external

187 morphology due to extensive wear but was later verified in thin section. Wear on the adult

188 mandible is evident on both the acrodont dentition and the jaw bone. Interestingly, the anterior

189 pleurodont dentition was mostly unworn in the adult specimen or worn to a minimal degree on

190 other adult specimens examined; this is similar to the condition seen in Agama agama, which has

191 been documented to replace its anterior pleurodont dentition (Cooper et al., 1970). However, the

192 anterior acrodont teeth are almost completly worn away, making it difficult to differentiate it

193 from the jaw bone; to circumvent this problem, tooth counts were made under a microscope and

194 later confirmed in thin section when possible. The wear observed on the anterior acrodont

195 dentition is extensive - often there were only traces of the tooth left - which is similar to the

196 dental wear seen in Uromastyx, a taxon that can become functionally edentulous in adulthood

197 (Throckmorton, 1979).

198 The evidence of extensive wear is not only seen on the teeth but is also found on the

199 mandible itself in the form of wear facets, which are only present on the labial side of the dentary 
200 where the maxillary dentition occludes, interdigitating between dentary tooth positions. Wear

201 facets have been characteristically found on the mandibles of acrodont squamates and

202 rhynchocephalians as far back as the Cretaceous (Simões et al., 2015), which are formed due to

203 the maxillary dentition wearing down on the dentary bone during mastication and passive

204 occlusion. These wear facets are present along the posterior two-thirds of the mandible but are

205 most developed in the posterior region of the dentary of adult specimens of $P$. vitticeps.

206 However, in adult individuals of other acrodontians, like chameleons (Dosedělová et al., 2016a),

207 the wear facets are found along the entire dentary. Lastly, it is important to mention that the jaw 208 ontogenetically increases posteriorly in length and dorsoventrally in width, with the articular

209 increasing in robustness, and the coronoid process moving posteriorly relative to the tooth row.

210 This ontogenetic change effectively creates more space for the posterior addition of teeth to the

211 dentary, a feature that is frequently seen in lepidosaurs (Berkovitz and Shellis, 2017 and

212 referances therein).

213 Histology

214 Juvenile tissue histology

215 Longitudinal and coronal sections of the mandible of the juvenile specimen (ROM

216 R8234) were examined (Fig. 2\&3). The mandibular ramus in the juvenile specimen is not as

217 well ossified as in the adult (ROM R8507) and has a medial curvature anteriorly; this made

218 obtaining sections of the anterior and posterior dentition within the same sectioning plane

219 difficult. Furthermore, the focus of this study is the non-replacing acrodont dentition, and

220 therefore, the anterior pleurodont dentition were not included in this study. In longitudinal

221 section, only the dentine is exposed with no other dental tissues (e.g., cementum, enamel) being

222 visible, this is likely due to sectioning bias. In longitudinal section, the implantation relationship

223 of the teeth to the jawbone is not easily discernable. However, this plane of section allows for

224 visualization of the incremental maturation of dentition. The most anterior teeth are the oldest, a

225 characterization based on the thickness of dentine, whilst the most posterior teeth are the

226 youngest and have the least amount of dentine infilling. In longitudinal section, the interaction

227 between the individual tooth and its neighboring teeth can be seen in this plane (Fig. 2B-D);

228 there is distinct tissue that attaches the teeth to each other as well as to the jaw bone. This tissue 
229 is tentatively identified as alveolar bone (sensu LeBlanc and Reisz, 2015) based on the position

230 and presumed function of the tissue rather than on its histological appearance, which does not

231 completely conform to alveolar bone description in literature. The alveolar bone in this specimen

232 has a woven appearance that is less organized than the bone that makes up the dentary. However,

233 it lacks the extensive trabecular or 'spongy' appearance that is found in other squamates, such as

234 snakes (Budney, 2004; Budney et al., 2006). This tissue attaches the teeth to each other, as well

235 as to the jaw bone proper, a function that has been attributed to 'bone of attachment' (Ananjeva

236 and Smirina, 2007), better known as alveolar bone (Budney et al., 2006; Caldwell, 2007;

237 LeBlanc and Reisz, 2013, 2015; LeBlanc et al., 2016a). The alveolar bone in P. vitticeps is also

238 not distinguished from the jaw bone by a reversal line; this is possibly an effect of the plane of

239 section or due to a lack of resorption prior to attachment (Fig. 2). It is important to note that not

240 all the teeth in the juvenile specimen are fully ankylosed to the dentary (Fig. 3); however, each

241 tooth seems to ossify to the adjacent dentition, which occurs prior to full ankylosis and which

242 mirrors what has been documented in Chamaeleo calyptratus (Buchtová et al., 2013; Dosedělová

243 et al., 2016a). This observation is based on the youngest tooth (Fig.3), which has yet to ankylose

244 to the dentary, which is attached to the neighboring teeth.

In coronal section, all of the common dental tissues are identifiable, with dentine comprising the bulk of the tooth identified by its characteristic radiating dentinal tubules. The enamel is best visualized in cross-polarized light and is unworn in the youngest teeth (Fig.4B). Because of the mediolateral compression of the tooth, the coronal plane of section is the best plane in which to examine the enamel as it transects the labial and lingual surfaces of the tooth this. The enamel is fairly thick, which has also been reported in Uromastyx (Throckmorton, 1979). Due to the lack of wear in the juvenile specimen, the enamel is of equal thickness on the lingual and labial sides of the tooth crown (Fig.4B). On the lingual side of the tooth, the tapering edge of the enamel leads down to a layer of acellular cementum, meeting at the cementoenamel junction, which defines the boundary between the anatomical crown and root of the tooth. It is likely that the cementum cannot be visualized on the labial side because the alveolar bone has grown to meet the cementum, effectively ankylosing the tooth while obstructing the cementum, or possibly because of resorption of the cementum in this process, although the latter has not been previously documented. 
In the coronal section of the juvenile specimen of $P$. vitticeps, it is evident that both the

260 shape of the dentition and the manner in which the tooth is implanted are more congruent with

261 the pleurodont condition, as the lingual side of the tooth is markedly greater in length than that of

262 the labial side (Fig. 4A, C). Although both ends of the tooth are ankylosed to the jaw, it is clear

263 that there is more contact with the jaw bone and more attachment tissues on the labial side, a

264 character of pleurodont implantation. Apart from the obvious labial bias of attachment, alveolar

265 bone can be seen at the base of the tooth, being more woven than the rest of the jaw bone yet

266 apparently lacking the more porous structure that is usually associated with alveolar bone. In

267 coronal section (Fig. 4B), the reversal line defining the boundary between the new alveolar bone

268 and the preexisting jaw bone is visible in cross-polarized light. The labial side of the tooth (Fig.

269 4C) is shorter and is attached to the jaw bone with more attachment tissue than that present at

270 lingual side; furthermore, the labial side appears to be actively remodeled by osteoblasts and

271 osteoclasts (Fig. 4D). The osteoclast on the labial side of the tooth is identified on the basis of its

272 large size, general shape and the resorption bay that is created in the dentine (Witten and

273 Huysseune, 2009). The high density of bone cell lacunae directly posterior to the osteoclast are

274 identified as osteocytes, which were osteoblasts, indicating that the labial side of the tooth is

275 being resorbed and that bone is being deposited in its place. It is also important to note that the

276 strut like structure of the cancellous bone likely give the mandible form and function prior to full

277 ossification and maturity.

Lastly, a coronal section through the coronoid process (Fig. 4E), shows the presence of a 279 developing tooth. Its identification as a developing tooth is based on its general shape, location 280 within the jaw, and the presence of thick enamel relative to the amount of dentine. The presence 281 of more enamel than dentine denotes an early stage of tooth development, as dentine is deposited 282 by odontoblasts later in development (Erickson, 1996; LeBlanc et al., 2016b). The presence of 283 this developing tooth was serendipitously discovered when sectioning the specimen, and there 284 were no external indicators that this tooth was buried within the bone of the coronoid process.

285 This new tooth was developing within the coronoid process and was visibly resorbing the bone 286 tissues of both the coronoid and the dentary, effectively making space for itself prior to 287 attachment.

288 Adult tissue histology 
In the longitudinal and coronal sections of the mandible of the adult specimen (ROM

290

291

292

293

294

295

296

297

298

299

300

301

302

303

304

305

306

307

308

309

310

311

312

313

314

315

316

317

318

319 R8057), lamellar bone makes up the main body of the dentary (Fig. 5). In coronal section, it can be seen that the large trabeculae seen in the juvenile have been incrementally infilled by lamellar bone, giving the mandible an osteological density that was not present in early ontogeny (Fig. 6). As previously reported in agamids (Ananjeva and Smirina, 2007), the bone tissue is not highly vascularized nor actively remodeled, particularly in dentary bone. This is in line with the findings in the adult jaw, where no reversal lines or large areas of remodeling are found. No primary or secondary osteons can be identified in the longitudinal sections. In longitudinal section (Fig. 5C), posterior to the most worn dentition, the wear facets become a marked feature of the dentary's labial surface. The pervasiveness and the depth of the wear facets should not be considered informative in thin section, as the variation of depth is a false impression, and is due to the sectioning plane in combination with the curvature of the dentary. The wear facets form pseudo-pedicels for the tooth remnants; these should not be confused for the 'bony pedicles' that have been identified developmentally in chameleons (Buchtová et al., 2013). The 'bony pedicles' in chamelons are formed by bone growing upwards to meet the developing tooth whilst the pseudo-pedicels in P.vitticeps are formed by the wear facets on the mandible, as the maxillary teeth wear away lamellar bone between the functional dentition on the jaw, leaving the remaining tooth caps on secondarily formed pedicels of lamellar bone (Fig. 5C-D). This gives the tooth implantation region its distinctly acrodont appearance.

The acrodont teeth in the adult specimen are markedly different than those described in the juvenile (Fig. 6). The unequal shape and implantation of the teeth reported in the juvenile is not recognized in the adult, which has acrodont implantation with no lingual bias. The anterior pleurodont dentition is shown to maintain its vasculature, which is associated with an open pulp cavity; this is typical of pleurodont dentition among squamates. Perhaps the most interesting feature of the longitudinal sections is seen in the worn acrodont teeth directly posterior to the pleurodont pair (Fig. 5). The teeth are worn to such an extent that often little dentine and no enamel is detectable (Fig. 5B), even in thin section. However, the vasculature is maintained and denotes tooth positions, and the pulp cavity remains open and vascularized throughout the functional life of the tooth (Fig. 5\&6). Thus, the teeth appear to remain viable into adulthood, which is in contrast to the condition reported in Chameleo (Dosedělová et al., 2016a) and in Uromastyx (Throckmorton, 1979). 

important to characterize the tissues involved. In the adult specimen, the tissues are distinct between the tooth and the platform of lamellar bone. The presence of these tissues alone provides evidence of remodeling, as they are not seen in the juvenile specimen (Fig. 4) and some tissue which were present no longer are identifiable in the adults (Fig. 6) In this case, 'remodeling' is used in the broad sense rather than to refer strictly to bone remodeling. For example, in this agamid, the dentine that was once present in the lingual side of the tooth of the juvenile has been resorbed and is no longer present, or it is present in a greatly reduced extent in the adult. In both instances, it has been replaced by woven bone. Positioned between the dentine and the organized lamellar bone of the jaw is a layer of woven bone can be seen at the base of the teeth in longitudinal section (Fig. 5). The woven appearance under cross-polarized light identifies this as woven bone, which is characterized by the unorganized fibers (Padin \&Lamm 2013) (Fig.6) the woven bone lacks vascular spaces, which are also mostly missing in the lamellar bone. The lack of organization in this bone indicates the occurrence of relatively fast deposition and is unlikely to be the original alveolar bone that was identified in the juvenile specimen (Hernandez et al., 2004). The true extent of remodeling and change in tooth morphology is best seen in crosspolarized light in coronal section as this plane allows for the best direct comparison to the juvenile teeth in Figure 4. In conjunction with its position relative to the teeth, it is tentatively identified here as a remodeling zone (sensu Budney, 2004) (Fig. 5B,6A). In coronal section (Fig. $6 \mathrm{~A}, \mathrm{C})$ the remodeling zone is clearly identifiable in cross-polarized light, and the distribution of dental tissues and bone is markedly different than that previously described in the juvenile specimen.

In the adult specimen, the anterior acrodont dentition was either completely worn away, or all that remained was a dentine fragment (Fig. 5). The most posterior dentition retained more comparatively more dentine and often with a lingual bias (Fig. 6B), but this was only discernable in thin section. The anterior acrodont teeth in the adult had no evidence of enamel, while the posterior most teeth were the only ones that maintained some enamel, although it is only present on the lingual side. Within the pulp cavity of the adult, there often remains evidence of vasculature (Fig. 6) although it is greatly reduced to the center of the tooth. The overall shape of the tooth is markedly different to that reported in the juvenile, and the external appearance is acrodont like that described in literature (e.g., Budney, 2004). 
Tooth replacement has been a topic of great interest in recent years in groups ranging

353 from fish to tetrapods and across broad time scales. There have been several studies documenting

354 the presence of replacement patterns in relation to dental development (e.g., Westergaard and

355 Ferguson, 1990; Richman and Handrigan, 2011; LeBlanc and Reisz, 2015). However, there has

356 been relatively little work on the anomalous squamates that completely cease tooth replacement,

357 the acrodontians. Other than mammals, most fish (but see ratfish, e.g., Huber et al., 2008),

358 amphibians, reptiles and non-mammalian synapsids have continuous tooth replacement through

359 life, making acrodontian squamates an anomaly amongst toothed vertebrates. The lack of

360 replacement comes with a set of challenges, two of which are addressed here. The first challenge

361 is how does the jaw grow whilst having permanently ankylosed dentition and whilst maintaining

362 occlusion with the maxillary dentition? The second challenge is how to maintain a single set of

363 functional teeth through the lifetime of an animal, which essentially is a problem of combating or

364 adapting to wear. These two obstacles are also faced by mammals, which have one primary set of

365 teeth throughout most of their life. In mammals, the issue of maintaining occlusion appears to be

366 solved by maintaining a ligamentous tooth attachment, which allows for the teeth to remain

367 mobile as the mandible grows and remodels (Lumsden and Osborn, 1977; LeBlanc and Reisz,

368 2013). The issue of wear is at least partially addressed by having much thicker prismatic enamel

369 than that found in most reptiles (Dauphin and Williams, 2008; Kieser et al., 2009). However, the

370 question remains how reptiles that permanently ankylose their dentition to the jaw and those with

371 reptilian enamel adapt to growth and wear.

372

373

374

375

376

377

378

379

\section{Wear adaptations}

This study found that the unworn teeth on the mandible of the juvenile agamid $P$.

vitticeps have a uniform layer of enamel on both the lingual and labial sides, and the enamel is relatively thick in comparison to polyphyodont reptiles such as crocodilians. The thick enamel also seems to occur in two other acrodontians, Uromastyx enamel has been documented as 'thickened' (Cooper and Poole, 1973; Throckmorton, 1979), and chameleons' enamel appears thick in recent studies but is not explicitly commented on (Buchtová et al., 2013; Dosedělová et al., 2016b). Furthermore, Uromastyx, a herbivorous acrodontian, has been reported to have 
380 thickened prismatic enamel (Throckmorton, 1979), similar to mammalian enamel. This shows

381 that thickening of the enamel is a convergent adaption against wear in both squamates and in 382 mammals.

Enamel thickening is not the only adaptation that acrodontians appear to have evolved in

384

385

386

387

388

389

390

391

392

393

394

395

396

397

398

399

400

401

402

403

404

405

406

407

408

409 order to combat wear. Previous studies of chameleons and Uromastyx have shown that their pulp cavities were infilled with 'mineralized tissue' (Dosedělová et al., 2016b) or 'bone' (Throckmorton, 1979). The likely purpose of this infilling is to prevent the pulp cavity from being exposed as the external surface of the tooth is worn away; both aforementioned studies also reported the disappearance or significant restriction of vasculature that initially supplied the dentition in early ontogeny. Dosedělová et al. (2016a) also showed increased mineralization in the bone underlying the tooth-bone junction in chameleons, and Throckmorton (1979) found that in Uromastyx, the bone below the teeth had become more compact in appearance rather than cancellous. These findings are quite comparable with one another, even though Uromastyx and chameleons are on two disparate branches of Acrodonta (Pyron et al., 2013). These results then beg the question: is this pattern more widespread across acrodontians?

This study found comparable results in Pogona vitticeps. The pulp cavity is also greatly diminished through ontogeny but is not completely 'obliterated,' as seen in Uromastyx. The infilling of the pulp cavity in $P$. vitticeps is done through a combination of dentine, and the bone. The progressive infilling of dentine is a normal development for teeth, as odontoblasts continue to sequentially deposit dentine post- eruption. What is abnormal is the bone growing into the pulp cavity and the resorption of the dentine root. In some of the sampled sections of $P$. vitticeps, the teeth fail to show the extent of bone infilling reported in Uromastyx, as the matrix that infills the pulp cavity does not seem to have any of the cellular spaces reported in Uromastyx. However, in other sections of the same individuals, the teeth experience a lot of remodeling and infilling, often with bone growing over the dentition. This is identified as 'bone' rather than 'mineralized material' as mentioned by (Dosedělová et al. 2016) based on the amount of osteocyte lacunae present as well as the presence of osteons. Together the bone and dentine restrict the pulp cavity to such an extent that the tooth can then be worn away without the risk of exposing the vulnerable vasculature and nerves that are within the pulp cavity. Furthermore, the bone making up the dentary is not vascularized and sequentially also becomes more infilled with 
410 bone, making compact bone through ontogeny; this allows for the jaw to be worn in the form of 411 distinct mandibular wear facets.

412 The amount of wear on the adult dentition of Pogona vitticeps changes the external 413 morphology significantly. This is common in ungulate mammals (Fortelius and Solounias, 414 2000;Kaiser et al.,2013;) and in herbivorous fossil reptiles (e.g., Reisz, 2006) but has not been

415 reported in other squamates. The dentine and enamel on the labial side of the mandible are worn 416 in the older specimens of $P$. vitticeps, which is compatible with the findings in Uromastyx 417 (Throckmorton, 1979), where depending on the tooth position, the enamel and dentine were 418 either completely worn away or were worn to such a degree that the lingual side of the tooth 419 retained a much thicker layer of these two tissues. This pattern is likely caused by the 420 combination of extensive wear from feeding and passive occlusion with the maxillary dentition, 421 as well as the complete lack of tooth replacement. Interestingly, the vasculature that leads to the 422 pulp cavities is still present in the adult specimens of Pogona vitticeps indicating that even the 423 most worn teeth probably remained viable through the heavy wear process and likely closed the 424 pulp cavity progressively until they were worn away. This phenomena has also been observed in 425 hadrosaurs, which progressively infill their dentition with dentine as it nears the occlusal surface 426 in their dental batteries and then wear away the entire tooth (LeBlanc et al., 2016b; Bramble et 427 al., 2017).

428 430 431 432 433 434 435 436 437 438

\section{Maintaining occlusion}

Continuous growth of the mandible throughout ontogeny is concurrent with the increased size of the skull. In most polyphyodont taxa, this is not problematic, as constant tooth replacement adjusts for increase in tooth size as well as possible migration (e.g., Haridy et al. 2018); even in mammals, after the aquasition of their permanent set of dentition, a maintained ligamentous attachment of teeth allows for migration and remodeling. However, for acrodont squamates that have ankylosed monophyodont dentition, this becomes problematic - the question becomes how occlusion is maintained as the jaw grows. Through examination of 37 specimens and histological sections, this study of $P$. vitticeps has shown that the growth of the jaw and the initiation of additional tooth development are decoupled processes. This is evident in the juvenile specimen in which the dentition is growing into the coronoid process (Fig. 3B, 
439 4E,F), where the youngest un-erupted tooth is resorbing the ventral portion of the coronoid 440 process in order to continue developing. This indicates that in the early stages of ontogeny, tooth 441 development likely happens at a rate faster than dentary growth. This is reinforced by comparing 442 the juvenile specimen (103mm SV length), which has 16 tooth positions, with the adult, which is 443 more than twice as long (222 mm SV length), only has 17 tooth positions. This supports the 444 hypothesis that attaining the maximum number of teeth occurs relatively early in ontogeny, well

445 before attaining maximum adult size. Tooth development eventually slows and likely stops early 446 in ontogeny, while the various jaw elements continue to grow. The mandible of $P$. vitticeps 447 undergoes many changes through ontogeny, but there is little distortion of the tooth row during 448 growth. This is likely achieved by appositional bone growth of the jaw, with deposition of 449 parallel-fibered bone both internally and externally to achieve the increase in internal 450 ossification, width and length that is seen in the adult and without remodeling or migration of the 451 ankylosed dentition. Through the histological results of this study, which show little bone 452 remodeling, and external observation of 37 specimens (see Table1 in supplementary material), it 453 is hypothesized that occlusion is maintained through the allometric growth of the various 454 portions of the jaw elements. The dentary and its corresponding dentition are the first to reach 455 adult size, while the other jaw elements continue to grow through ontogeny, gradually increasing 456 in robustness but not interfering with occlusion.

Acrodont implantation as a result of ontogenetic remodeling

Acrodont implantation is defined ambiguously and inconsistently in literature. The mode of implantation has been defined as attachment to the 'edge' of a jaw (Peyer, 1968), as teeth ankylosed to the 'apex' of the jaw by cement (Edmund 1969), or as the fusion of dentition to the 'margin' of the jaw (Motani, 1997). Eventually, the lack of replacement also became a character of acrodont tooth implantation (Zaher and Rippel, 1999), as the condition is present in acrodont squamates. Implantation categories are pervasive in literature and are often used as phylogenetic characters, but these definitions are problematic and tend to imply an evolutionary progression of tooth implantation from simple to more complex (see full implantation review in: Budney et al., 2006). In reality, the traditional categories of acrodonty, pleurodonty, and thecodonty are, at best, descriptive terms (Estes and Charles, 1988) (Fig. 7A). Pogona vitticeps and likely other agamids change implantation categories through ontogeny, and therefore, they are a good representation 
469 as to why implantation categories should only be used descriptively. The teeth in the juvenile $P$.

470 vitticeps are unequal in shape, with the lingual side being much longer than that of the labial side

471 (Fig. 4), and attachment mostly occurring on the labial side, which are characteristics of

472 pleurodont implantation. Traditionally in literature, the anatomy present in adult individuals of

473 various taxa is described; this likely explains why acrodont squamates got their namesake since

474 in their adult stage, the teeth do appear acrodont. This study reveals how implantation may

475 appear to be acrodont in adulthood through a combination of factors (Fig 7); (1) the gradual

476 infilling of teeth with dentine that allows for progressive wear (2) resorption of the dentine by

477 odontoclasts (Fig4C,D), (3) growth on the dentary that infills part of the pulp cavity with woven

478 bone and that reduces the trabecular structure of the dentary bone, (4) the continued remodeling

479 of the dentary which removes traces of buried dentine and the previous pleurodont dentition.

480 Tooth wear then further changes the crown morphology the maxillary dentition occludes with the

481 mandibular dentition wearing away the labial side of the mandibular dentition and wearing away

482 part of the dentary creating wear facets. This progression model shows how teeth that were

483 originally pleurodont in implantation can appear acrodont and have an entirely different

484 morphology due to a combination of dentine infilling, bone remodeling, and tooth wear. It is

485 difficult to ascertain without experimental testing if these secondary remodeling steps would

486 occur in any pleurodont squamate taxa but are suppressed by tooth replacement, or if these are

487 secondarily acquired adaptations in monophyodont taxa to combat wear. Therefore, at least in

488 the agamid $P$. vitticeps, the dentition changes from pleurodont to acrodont in appearance through

489 ontogeny. This indicates that we should not use implantation categories as phylogenetic

490 characters if the ontogenetic stage of the specimen is unknown, as is the case in many fossil taxa.

491 Conclusion

492

The dentition of acrodontians has recently been an area of interest in the context of dental

493 development and implantation, as this group is known for its mode of implantation and

494 monophyodonty. The latter is an anomalous occurrence among tetrapods, as intermittent to

495 continuous replacement of teeth is the primitive condition for toothed vertebrates, including most

496 extant fish, amphibians, and amniotes. This lack of replacement has become associated with

497 acrodonty, resulting in monophyodonty becoming a formal characteristic of acrodont

498 implantation in squamates, and previous authors have implied that acrodont implantation is the 
499 cause of monophyodonty. However, this study shows that at least some modern acrodont reptiles

500 do not initially have acrodont implantation early in ontogeny, and there is a distinct ontogenetic

501 change in the morphology and implantation of dentition of the agamid Pogona vitticeps. The

502 youngest teeth in juvenile specimens are pleurodont in implantation, with a greater lingual

503 contribution of tooth tissues and an attachment biased towards the labial side. These tissues are

504 secondarily remodeled through a step-wise process of: (1) resorption of dentine, (2) deposition of

505 bone and dentine, (3) wear of the tooth surface, and (4) wear of the jaw bone proper, effectively

506 changing the morphology. These processes give $P$. vitticeps the appearance of acrodont dentition

507 in adulthood. This is an important distinction to make as it signifies that this acrodont squamate,

508 and likely other acrodont reptiles, do not develop acrodont teeth, but rather develop pleurodont

509 teeth like the vast majority of squamates, making their teeth inherently pleurodont, and

510 secondarily acrodont. Therefore, the wear adaptations, remodeling, and dental wear come

511 together to give the appearance of an acrodont mode of implantation. The wear adaptations

512 documented in this study likely evolved due to the lack of replacement. Importantly the

513 monophyodont condition causes the acrodont condition, rather than acrodonty causing

514 monophyodonty, as has been implied in literature.

515 This raises the question as to how we should code acrodont dentition in squamate

516 phylogenies; is it true acrodonty if this implantation mode is only achieved through secondary

517 remodeling of the teeth and dentary? Finally, another important consideration, and a direction of

518 future studies, is that if all acrodontians share these ontogenetic changes and wear adaptations,

519 then what is the significance of the convergence seen between acrodontian squamates and the

520 rhynchocephalian Sphenodon, which is also reported to have acrodont dentition?

\section{Acknowledgments}

Incredible thanks go to Robert R. Reisz and Aaron H. Leblanc who brought this issue to

523 light and encouraged the development of this study. I would like to thank Diane Scott for

524 photographs and SEM images and bearded dragon husbandry advice. Thanks to Bryan Gee for

525 his everlasting patience and editing of this manuscript into presentable form. I would like to

526 thank Kevin Seymour of the Royal Ontario museum (ROM) for assistance in locating

527 appropriate specimens for this study and to thank David Evans for allowing the use of the ROM 
528 histology laboratory and reading early versions of this manuscript. Thanks goes to Megan

529 Whitney and Kelsey Jenkins for their helpful reviews which improved this manuscript. Lastly, I

530 would like to acknowledge Osiris the bearded dragon whose presence in the lab was essential to

531 the direction of this study.

\section{References}

533 Ananjeva, N., and E. Smirina. 2007. Growth layers in bones and acrodont teeth of the agamid lizard Laudakia stoliczkana (Blanford, 1875) (Agamidae, Sauria). Amphibia-Reptilia 28:193-204.

Benton, M. J. (1984). Tooth form, growth, and function in Triassic rhynchosaurs (Reptilia, Diapsida). Palaeontology, 27(Part 4), 737-776.

Berkovitz BK, Shellis RP. The teeth of non-mammalian vertebrates. Academic Press; 2016 Oct 14.

Bramble, K., A. R. H. LeBlanc, D. O. Lamoureux, M. Wosik, and P. J. Currie. 2017. Histological evidence for a dynamic dental battery in hadrosaurid dinosaurs. Scientific Reports 7:15787.

Brown, C. M., C. S. Vanburen, D. W. Larson, K. S. Brink, N. E. Campione, M. J. Vavrek, and D. C. Evans. 2015. Tooth counts through growth in diapsid reptiles: Implications for interpreting individual and size-related variation in the fossil record. Journal of Anatomy

Buchtová, M., O. Zahradníček, S. Balková, and A. S. Tucker. 2013. Odontogenesis in the Veiled

Caldwell, M. W. 2007. Ontogeny, anatomy and attachment of the dentition in mosasaurs (Mosasauridae: Squamata). Zoological Journal of the Linnean Society 149:687-700. reference to tooth replacement. Journal of Zoology, 162(1), 85-98. 
558 Cooper JS, Poole DF. 2009. The dentition and dental tissues of the agamid lizard, Uromastyx. 559 Journal of Zoology. 1973 Jan;169(1):85-100.

560 Cope, E. D. 1864. On the characters of the higher groups of Reptilia Squamata: and especially of 561 the Diploglossa. Academy of Natural Sciences 16:224-231.

562

563

564

565

566

567

568

569

570

571

572

573

574

575

576

577

578

579

580

581

582

583

584

585

586

587

588

Dauphin, Y., and C. T. Williams. 2008. Chemical composition of enamel and dentine in modern reptile teeth. Mineral Mag 72:247-250.

Delgado, S., T. Davit-Beal, and J. Y. Sire. 2003. Dentition and tooth replacement pattern in Chalcides (Squamata; Scincidae). Journal of Morphology 256:146-159.

Dosedělová, H., K. Štěpánková, T. Zikmund, H. Lesot, J. Kaiser, K. Novotný, J. Štembírek, Z. Knotek, O. Zahradníček, and M. Buchtová. 2016. Age-related changes in the tooth-bone interface area of acrodont dentition in the chameleon. Journal of Anatomy 229:356-368.

Edmund, A. G. (1960). Tooth replacement phenomena in the lower vertebrates. R. Ont. Mus. Life Sci., 52, 1-190.

Erickson, G. M. 1996. Incremental lines of von Ebner in dinosaurs and the assessment of tooth replacement rates using growth line counts. Proceedings of the National Academy of Sciences of the United States of America 93:14623-14627.

Estes, R., and C. Charles. 1988. Phylogenetic Relationships of the Lizard Families: Essays Commemorating Charles L. Camp, illustrate. (Gregory K. Pregill (ed.)). Stanford University Press, $631 \mathrm{pp}$.

Fortelius M, Solounias N. Functional characterization of ungulate molars using the abrasionattrition wear gradient: a new method for reconstructing paleodiets. American Museum Novitates. 2000 Oct:1-36.

García, R. A., and V. Zurriaguz. 2016. Histology of teeth and tooth attachment in titanosaurs (Dinosauria; Sauropoda). Cretaceous Research 57:248-256.

Haridy, Y., A. R. H. LeBlanc, and R. R. Reisz. 2018. The Permian reptile Opisthodontosaurus carrolli: a model for acrodont tooth replacement and dental ontogeny. Journal of Anatomy 232:371-382.

Hernandez, C. J., Majeska, R. J., \& Schaffler, M. B. 2004. Osteocyte density in woven bone. Bone, 35(5), 1095-1099.

Huber, D. R., M. N. Dean, and A. P. Summers. 2008. Hard prey, soft jaws and the ontogeny of feeding mechanics in the spotted ratfish Hydrolagus colliei. Journal of The Royal Society 
Interface 5:941-953.

590

591

592

593

594

595

596

597

598

599

600

601

602

603

604

605

606

607

608

609

610

611

612

613

614

615

616

617

618

Järvinen, E., M. Tummers, and I. Thesleff. 2009. The role of the dental lamina in mammalian tooth replacement. Journal of Experimental Zoology Part B: Molecular and Developmental Evolution 312:281-291.

Jenkins, K. M., M. E. H. Jones, T. Zikmund, A. Boyde, and J. D. Daza. 2017. A review of tooth implantation among rhynchocephalians (Lepidosauria). Journal of Herpetology 51:300-306.

Kaiser, T. M., Müller, D. W., Fortelius, M., Schulz, E., Codron, D., \& Clauss, M. 2013. Hypsodonty and tooth facet development in relation to diet and habitat in herbivorous ungulates: implications for understanding tooth wear. Mammal Review, 43(1), 34-46.

Kieser, J. A., T. Tkatchenko, M. C. Dean, M. E. H. Jones, W. Duncan, and N. J. Nelson. 2009. Microstructure of dental hard tissues and bone in the tuatara dentary, Sphenodon punctatus (Diapsida: Lepidosauria: Rhynchocephalia). Frontiers of Oral Biology 13:80-85.

Kline, A. L. W., and D. Cullum. 2017. A long term study of the tooth replacement phenomenon in the young green iguana, Iguana iguana. Society for the Study of Amphibians and Reptiles 18:176-185.

LeBlanc, A. R. H., and R. R. Reisz. 2013. Periodontal ligament, cementum, and alveolar bone in the oldest herbivorous tetrapods, and their evolutionary significance. PLoS ONE 8.

LeBlanc, A. R. H., and R. R. Reisz. 2015. Patterns of tooth development and replacement in captorhinid reptiles: a comparative approach for understanding the origin of multiple tooth rows. Journal of Vertebrate Paleontology 4634:e919928.

LeBlanc, A. R. H., R. R. Reisz, D. C. Evans, and A. M. Bailleul. 2016a. Ontogeny reveals function and evolution of the hadrosaurid dinosaur dental battery. BMC Evolutionary Biology 16:152.

LeBlanc, A. R. H., R. R. Reisz, K. S. Brink, and F. Abdala. 2016b. Mineralized periodontia in extinct relatives of mammals shed light on the evolutionary history of mineral homeostasis in periodontal tissue maintenance. Journal of Clinical Periodontology 43:323-332.

Lumsden, A. G. S., and J. W. Osborn. 1977. The evolution of chewing: A dentist's view of palaeontology. Journal of Dentistry 5:269-287.

Montanucci, R. 2008. Comparative dentition in four iguanid lizards. Herpetologica 24:305-315.

Motani, R. 1997. Temporal and Spatial Distribution of Tooth Implantation in Icthyosaurs; pp. 
Peyer, B. (1968). Comparative odontology. University of Chicago Press.

623

Pyron, R., F. T. Burbrink, and J. J. Wiens. 2013. A phylogeny and revised classification of Squamata, including 4161 species of lizards and snakes. BMC Evolutionary Biology 13:93.

625

Reisz RR. Origin of dental occlusion in tetrapods: signal for terrestrial vertebrate evolution?.

626 Journal of Experimental Zoology Part B: Molecular and Developmental Evolution. 2006 May 15;306(3):261-77.

Richman, J. M., and G. R. Handrigan. 2011. Reptilian tooth development. Genesis 49:247-260.

Shellis, R. P., and B. K. B. Berkovitz. 1976. Observations on the dental anatomy of piranhas (Characidae) with special reference to tooth structure. Journal of Zoology 180:69-84. stem acrodontan lizard in the Cretaceous of Brazil revises early lizard evolution in Gondwana. Nature Communications 6:8149.

Smirina, E., and N. Ananjeva. 2017. On the longevity, growth and reproductive characteristics of Lichtenstein's Toadhead Agama, Phrynocephalus interscapularis Lichtenstein, 1856 (Agamidae, Sauria). Amphibia-Reptilia 38:31-39.

Smirina, E. M., and N. B. Ananjeva. 2007. Growth layers in bones and acrodont teeth of the agamid lizard Laudakia stoliczkana (Blanford, 1875)(Agamidae, Sauria). AmphibiaReptilia 28:193-204. character?. Microscopy research and technique, 59(5), 408-434.

Spencer, M. A. 2005. Dental functional morphology: How teeth work. American Journal of Human Biology 17:384-385.

Throckmorton, G. S. 1979. The effect of wear on the cheek teeth and associated dental tissues of the lizard Uromastix aegyptius (Agamidae). Journal of Morphology 160:195-207. (Iguanidae) and Uromastix aegyptius (Agarnidae). Journal of Morphology 148:363-390.

648 Westergaard, B., and M. W. J. Ferguson. 1990. Development of the dentition in Alligator 649 mississippiensis: Upper jaw dental and craniofacial development in embryos, hatchlings, 
650

651

652

653

654

655

656

657

658

659

660

661

662

663

664

665

666

667

668

669

670

671

672

673

674

675

676

677

678

and young juveniles, with a comparison to lower jaw development. American Journal of Anatomy 187:393-421.

Witten, P. E., and A. Huysseune. 2009. A comparative view on mechanisms and functions of skeletal remodelling in teleost fish, with special emphasis on osteoclasts and their function. Biological Reviews 84:315-346.

Zaher, H., and O. Rippel. 1999. Tooth implantation and replacement in Squamates, with special reference to mosasaur lizards and snakes. American Museum Novitates 3271:1-19.

Zari, T. A. 1999. On the reproductive biology of the herbivorous spiny-tailed agamid Uromastyx philbyi in Western Saudi Arabia. Zoology in the Middle East 19:123-130.

\section{Captions}

FIGURE 1. A comparative figure showing the external morphological differences in the dentition and mandibles between juvenile and adult specimens of Pogona vitticeps; (A) Right mandibular ramus of juvenile specimen ROM R8234; (B) Right mandibular ramus of adult specimen ROM R8507;(C) Closeup of wear facets on dentary in adult P.vitticeps (D) superimposed outlines of mandibles of varying ontogenetic stages, showing that most growth occurs in the posterior end of the mandible. Abbreviations; ad, acrodont dentition; cp, coronoid process; pd, pleurodont dentition; wf, wear facets. Arrows indicating un-ankylosed teeth. Scale bar $=1 \mathrm{~cm}$.

FIGURE 2. Details of external morphology of the juvenile dentition of Pogona vitticeps. (A) ROM R8234 in lingual view showing un-ankylosed dentition, the most posterior growing into the coronoid; (B) ROM R8418 scanning electron microscope image of incomplete juvenile mandible with; (C) closeup showing fibrous alveolar bone between the teeth; (D) closeup showing the lingual contribution of dentine to the teeth; (E) closeup showing the youngest tooth growing into and resorbing the coronoid. Abbreviations; ab, location of alveolar bone; cp, coronoid process; de, dentine. Arrows showing un-ankylosed teeth.

FIGURE 3. Longitudinal histological sections of the juvenile mandible of Pogona vitticeps with a focus on the dentition. (A) External view of ROM R8234, box outlining the dentition cut; (B) a section of the posteriormost seven tooth positions. (C) a section of the posterior seven tooth 
679 positions in cross polarized light; (D) a schematic representation showing the distinct tissues as 680 well as the progressive ankylosis of the teeth. Abbreviations; ab, alveolar bone; de, dentine; lb, 681 lamallar bone; pc, pulp cavity. Red arrow indicates where the newest tooth is resorbing the 682 previous tooth. Scale bar $=1000$ um

683 FIGURE 4. Coronal sections of juvenile mandible of Pogona vitticeps with a special focus on 684 tooth histology. (A) Coronal section of the jaw and tooth of a juvenile specimen of $P$. vitticeps 685 ROM R8510 showing minimal ossification of the jaw bone, and pleurodont tooth attachment; 686 (B) close up of a juvenile specimen's (ROM R8510) dentition, showing unworn morphology; (C) 687 coronal section of a juvenile specimen (ROM R8234) showing pleurodont implantation, and 688 remodeling; (D) closeup of the attachment site in ROM R8234, showing the labial side of the 689 dentition being resorbed by an osteoclast; (E) coronal section of the coronoid process (ROM R8234) showing a tooth developing lingually inside the coronoid and dentary; (F) a close up of

691 the tooth in the jaw bone, showing the resorption of the jawbone around newly developing tooth.

692 Abbreviations: ab, alveolar bone; ac, acellular cementum; cb, cancellous bone; $\mathbf{c p}$, coronoid 693 process de, dentine; dt, developing tooth; lb, lamellar bone; en, enamel; ost, osteocyte lacunae; 694 oc, osteoclast; rb, resorption bay; rl, reversal line. All un-labeled scale bars $=1 \mathrm{~mm}$.

FIGURE 5. Longitudinal sections of adult mandible of Pogona vitticeps with a focus on tooth histology. (A) External view of specimen ROM R8507, box outlining the dentulous area sectioned; (B) broad view showing the variation between the anterior and the posterior dentition; (C) a closeup of the worn dentition showing wear facets and remodeling zones; (D) broad view showing the variation between the anterior and the posterior dentition, showing the depth of wear facets and arrangement of vasculature; (E) a close up of the anterior dentition with open pulp cavities and associated vasculature. Abbreviations: de, dentine; jb, jaw bone; pc, pulp cavity; pp, pseudo-pedicle; vc, vascular canal; wf, wear facet. The arrows demarcate the extensive vasculature leading to pulp cavities. Scale bar=1000um

704 FIGURE 6: Coronal sections of adult mandible of Pogona vitticeps with a focus on tooth histology. (A) Coronal section of the jaw and tooth of an adult specimen of $P$. vitticeps (ROM R8507) showing the maintained vasculature and worn enamel on the labial side, in cross 
708 the jaw and tooth of an adult specimen of $P$. vitticeps ROM R8507 showing the extensive

709 remodeling of the tooth attachment site as well as the tooth, in cross polarized light;(D) Coronal

710 section of ' $C$ ' in normal transmitted light. Abbreviations: de, dentine; lb, lamellar bone; en,

711 enamel; vc, vascular canals; rz, remodeling zone. Scale bar=500um

712 FIGURE 7: Schematic explanation of implantation changes through ontogeny and tooth wear.

713 (A) three basic traditional implantation categories, without attachment tissue illustrated; (B)

714 pleurodont impanated tooth is attached via alveolar bone, yellow arrows show direction of

715 dentine deposition; (C) purple arrows show direction of dentine resorption by odontoclasts, blue

716 arrow shows direction of bone deposition by odontoblasts; (D) blue arrow shows further bone

717 deposition, trabecula in the dentary is reduced; (E) the tooth appears implanted at the apex of the

718 dentary; (F) Shows the position of maxillary tooth; $(\mathrm{G})$ the enamel is worn on the labial side; $(\mathrm{H})$

719 with advanced wear, the enamel, dentine, and dentary bone are worn on the labial side.

720

721 Supplementary

722 S.FIGURE 1. Ontogenetic sequence of $P$. vitticeps mandibles that were used to make the growth 723 schematic in Figure 1C. (A) ROM R8234; (B) ROM R8150; (C) ROM R8227;(D) ROM R9422;

724 (E) ROM R8507; (F) ROM R8189. Scale bar = $1 \mathrm{~cm}$.

725 TABLE 1. A table of all specimens of $P$. vitticeps examined for this study. In orange are specific 726 specimens either sectioned or figured. 


\section{Figure 1}

A comparative figure showing the external morphological differences in the dentition and mandibles between juvenile and adult specimens of Pogona vitticeps

(A) Right mandibular ramus of juvenile specimen ROM R8234; (B) Right mandibular ramus of adult specimen ROM R8507;(C) Closeup of wear facets on dentary in adult P.vitticeps (D) superimposed outlines of mandibles of varying ontogenetic stages, showing that most growth occurs in the posterior end of the mandible. Abbreviations; ad, acrodont dentition; $\mathbf{c p}$, coronoid process; pd, pleurodont dentition; wf, wear facets. Arrows indicating un-ankylosed teeth. Scale bar $=1 \mathrm{~cm}$. 
A

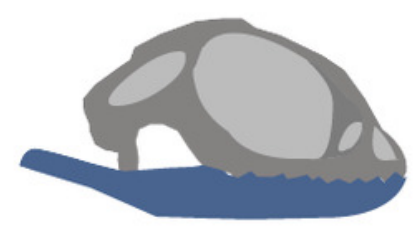

cpA $\quad$ ad $p d$

B
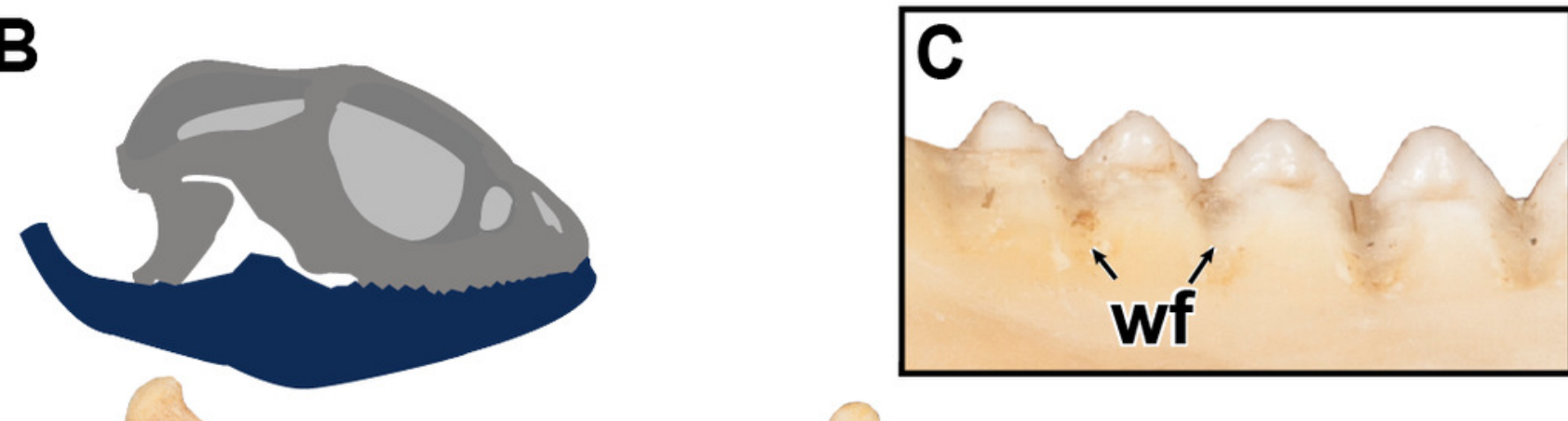

$c p$

ad

pd

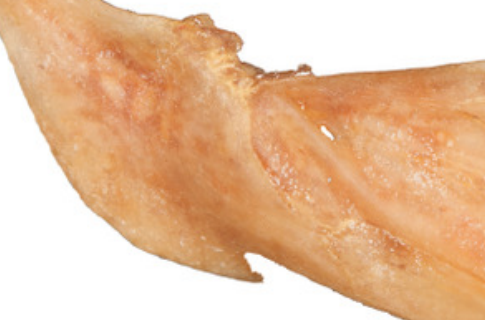

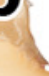

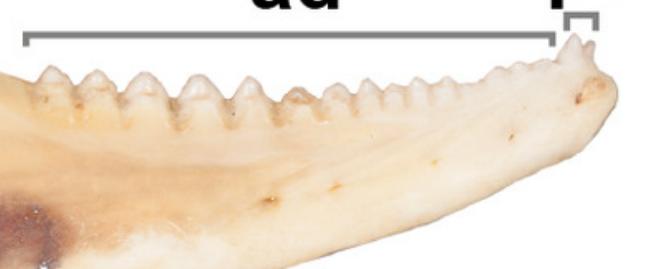

D

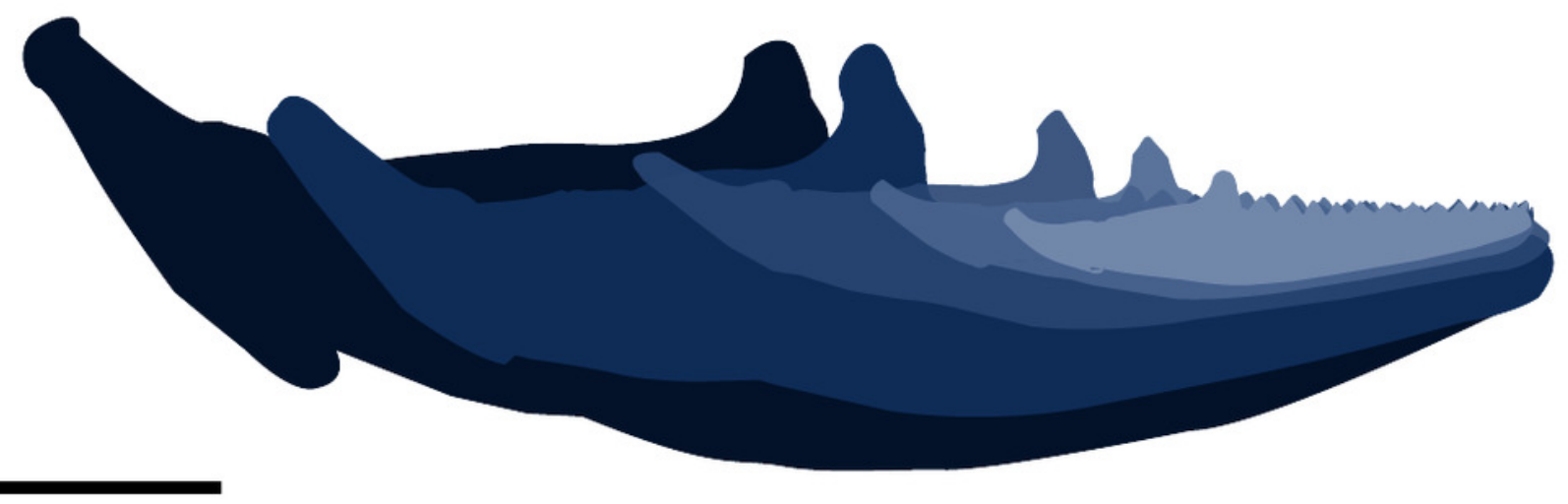




\section{Figure 2}

Details of external morphology of the juvenile dentition of Pogona vitticeps.

(A) ROM R8234 in lingual view showing un-ankylosed dentition, the most posterior growing into the coronoid; (B) ROM R8418 scanning electron microscope image of incomplete juvenile mandible with; (C) closeup showing fibrous alveolar bone between the teeth; (D) closeup showing the lingual contribution of dentine to the teeth; $(E)$ closeup showing the youngest tooth growing into and resorbing the coronoid. Abbreviations; ab, location of alveolar bone; $\mathbf{c p}$, coronoid process; de, dentine. Arrows showing un-ankylosed teeth. 


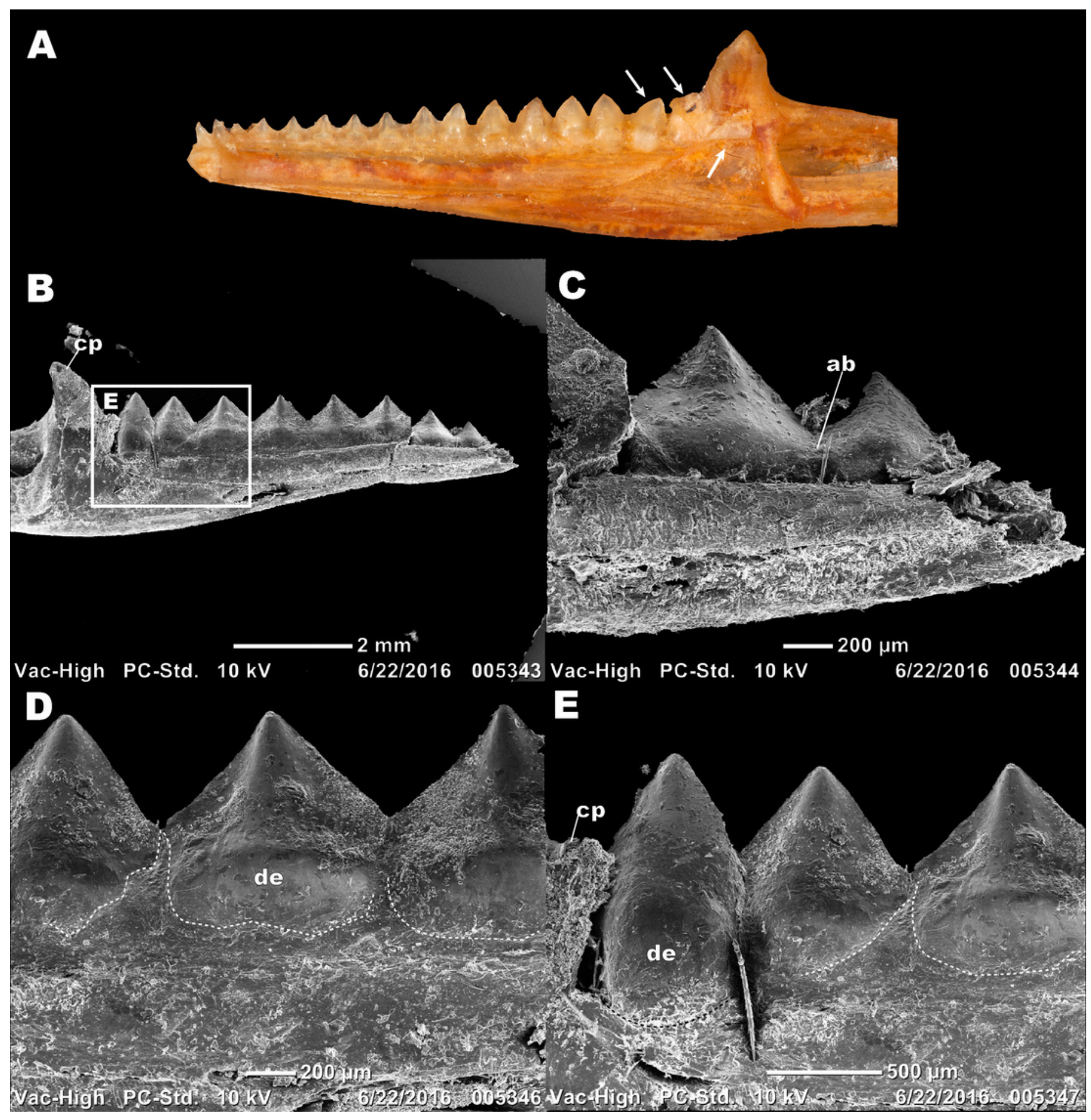




\section{Figure 3}

Longitudinal histological sections of the juvenile mandible of Pogona vitticeps with a focus on the dentition.

(A) External view of ROM R8234, box outlining the dentition cut; (B) a section of the posteriormost seven tooth positions. (C) a section of the posterior seven tooth positions in cross polarized light; (D) a schematic representation showing the distinct tissues as well as the progressive ankylosis of the teeth. Abbreviations; ab, alveolar bone; de, dentine; lb, lamallar bone; pc, pulp cavity. Red arrow indicates where the newest tooth is resorbing the previous tooth. Scale bar $=1000$ um 
A
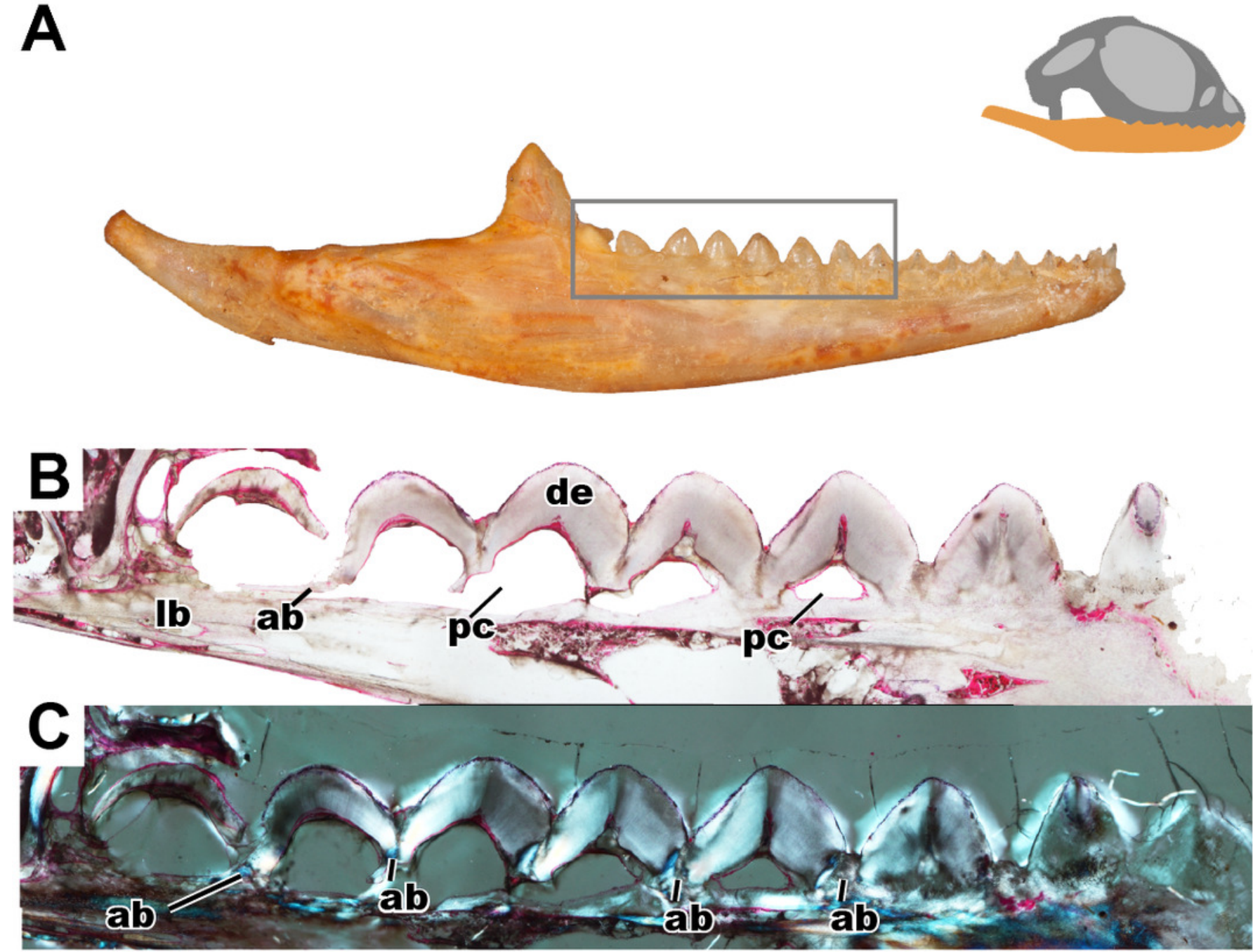

D

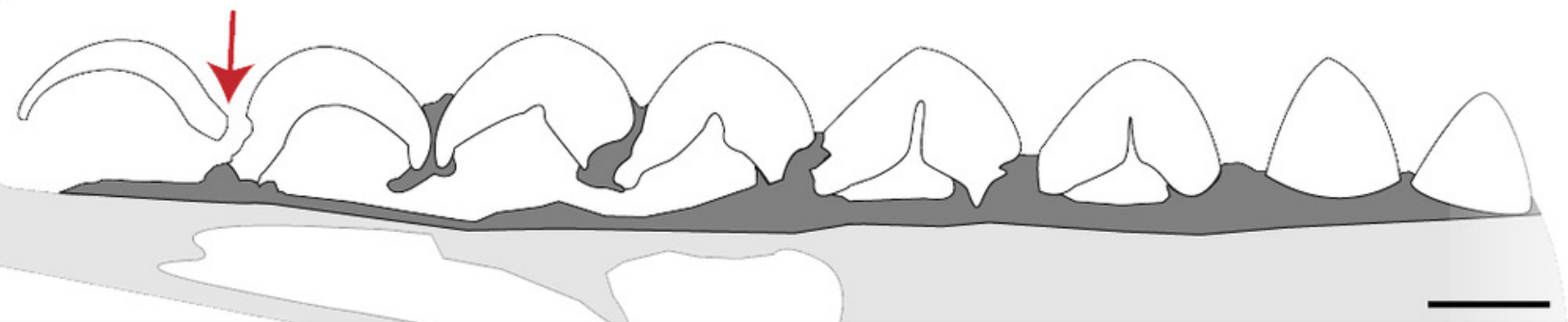

Lamellar bone $\square$ Alveolar bone $\square$ Dentition 


\section{Figure 4}

Coronal sections of juvenile mandible of Pogona vitticeps with a special focus on tooth histology.

(A) Coronal section of the jaw and tooth of a juvenile specimen of $P$. vitticeps ROM R8510 showing minimal ossification of the jaw bone, and pleurodont tooth attachment; (B) close up of a juvenile specimen's (ROM R8510) dentition, showing unworn morphology; (C) coronal section of a juvenile specimen (ROM R8234) showing pleurodont implantation, and remodeling; (D) closeup of the attachment site in ROM R8234, showing the labial side of the dentition being resorbed by an osteoclast; (E) coronal section of the coronoid process (ROM R8234) showing a tooth developing lingually inside the coronoid and dentary; (F) a close up of the tooth in the jaw bone, showing the resorption of the jawbone around newly developing tooth. Abbreviations: ab, alveolar bone; ac, acellular cementum; cb, cancellous bone; $\mathbf{c p}$, coronoid process de, dentine; dt, developing tooth; lb, lamellar bone; en, enamel; ost, osteocyte lacunae; oc, osteoclast; rb, resorption bay; rl, reversal line. All un-labeled scale bars $=1 \mathrm{~mm}$. 

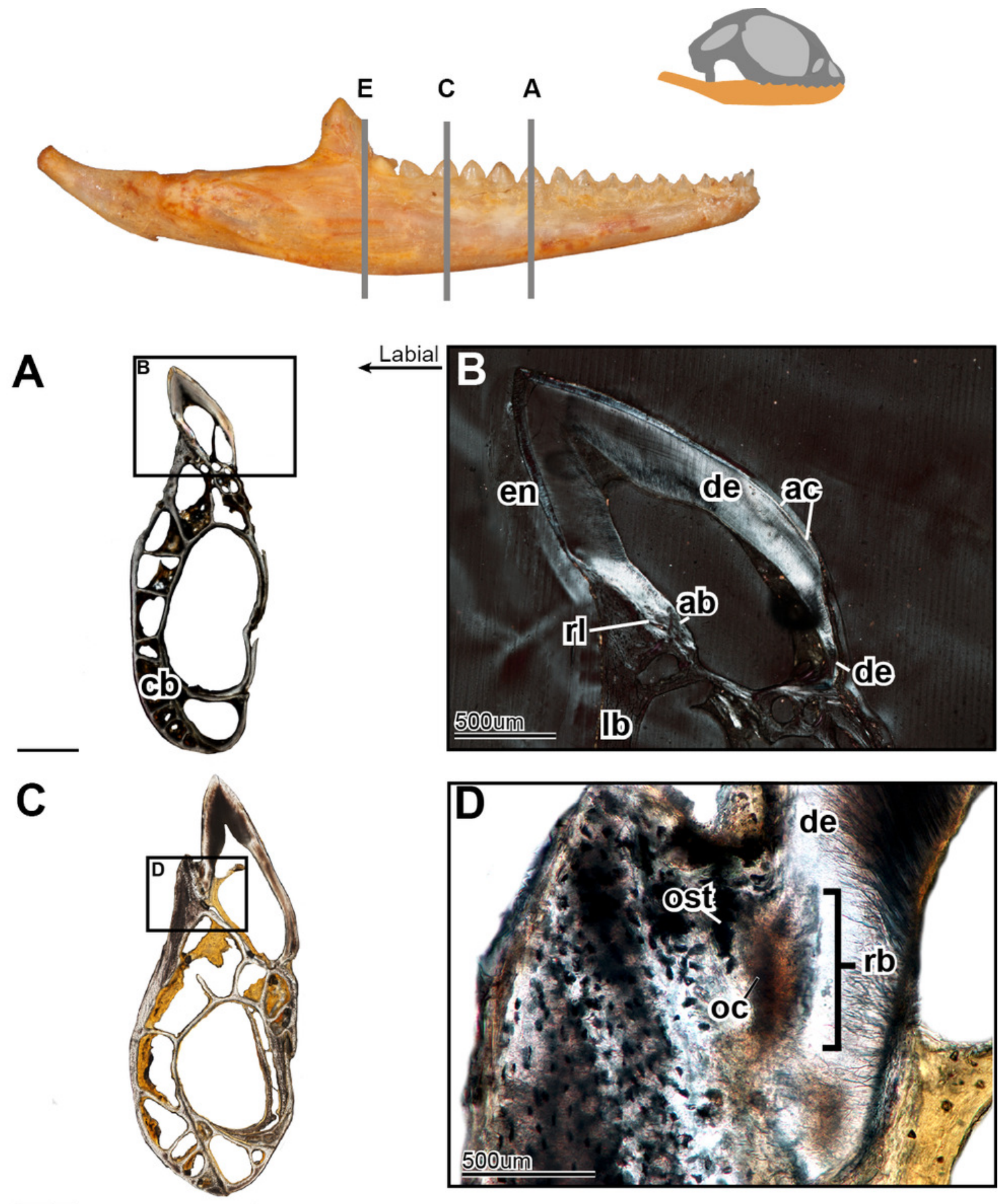

$\mathbf{E}$
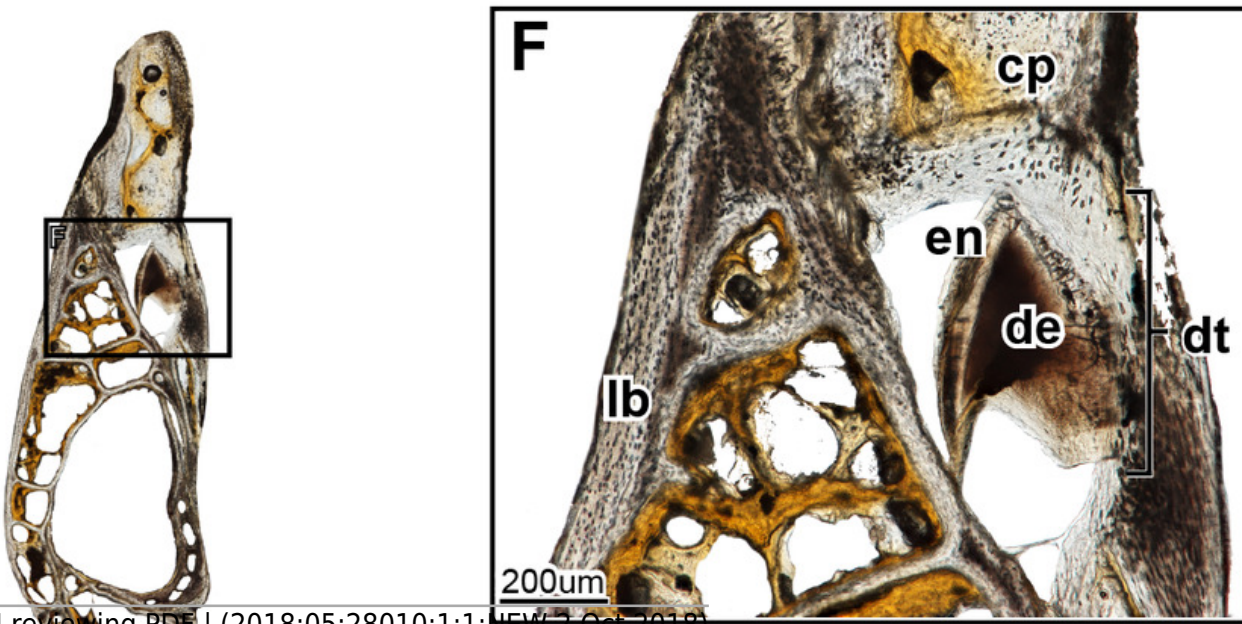


\section{Figure 5}

Longitudinal sections of adult mandible of Pogona vitticeps with a focus on tooth histology.

(A) External view of specimen ROM R8507, box outlining the dentulous area sectioned; (B) broad view showing the variation between the anterior and the posterior dentition; (C) a closeup of the worn dentition showing wear facets and remodeling zones; (D) broad view showing the variation between the anterior and the posterior dentition, showing the depth of wear facets and arrangement of vasculature; $(E)$ a close up of the anterior dentition with open pulp cavities and associated vasculature. Abbreviations: de, dentine; jb, jaw bone; pc, pulp cavity; pp, pseudo-pedicle; vc, vascular canal; wf, wear facet. The arrows demarcate the extensive vasculature leading to pulp cavities. Scale bar=1000um 

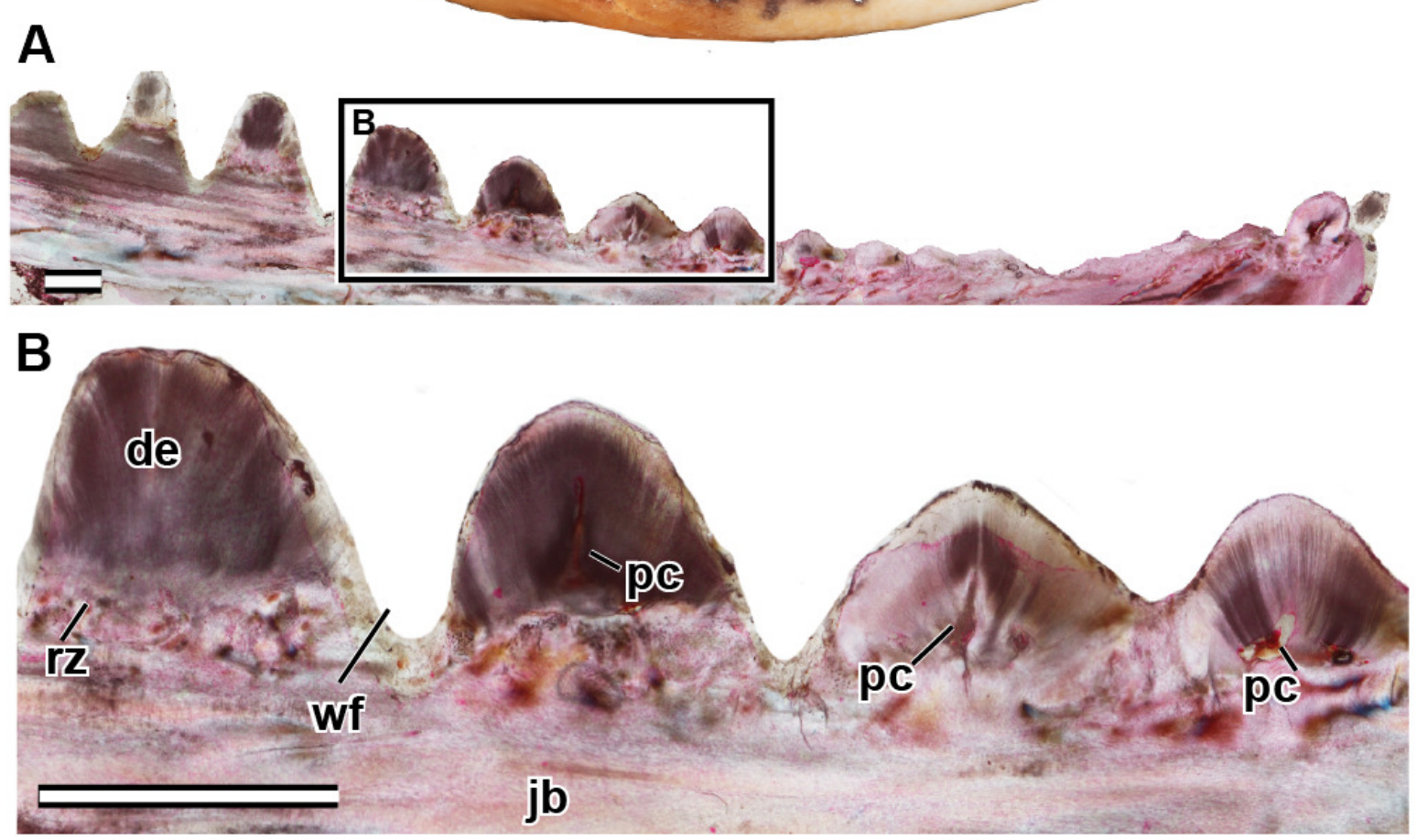

C
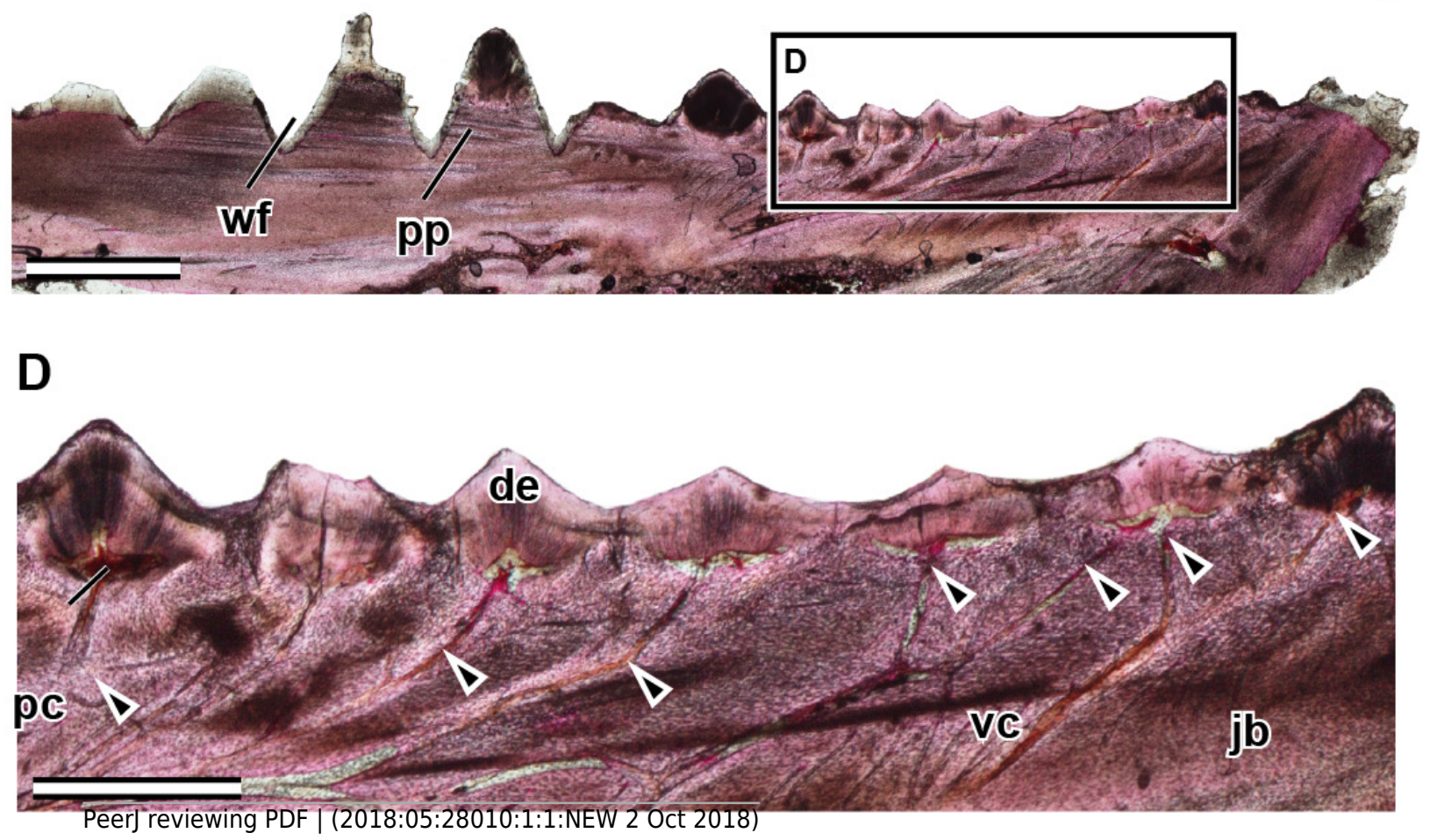


\section{Figure 6}

Coronal sections of adult mandible of Pogona vitticeps with a focus on tooth histology.

(A) Coronal section of the jaw and tooth of an adult specimen of $P$. vitticeps (ROM R8507) showing the maintained vasculature and worn enamel on the labial side, in cross polarized light; (B) Coronal section of ' $A$ ' in normal transmitted light; (C) Coronal section of the jaw and tooth of an adult specimen of $P$. vitticeps ROM R8507 showing the extensive remodeling of the tooth attachment site as well as the tooth, in cross polarized light;(D) Coronal section of ' $C$ ' in normal transmitted light. Abbreviations: de, dentine; Ib, lamellar bone; en, enamel; vc, vascular canals; rz, remodeling zone. Scale bar $=500$ um 

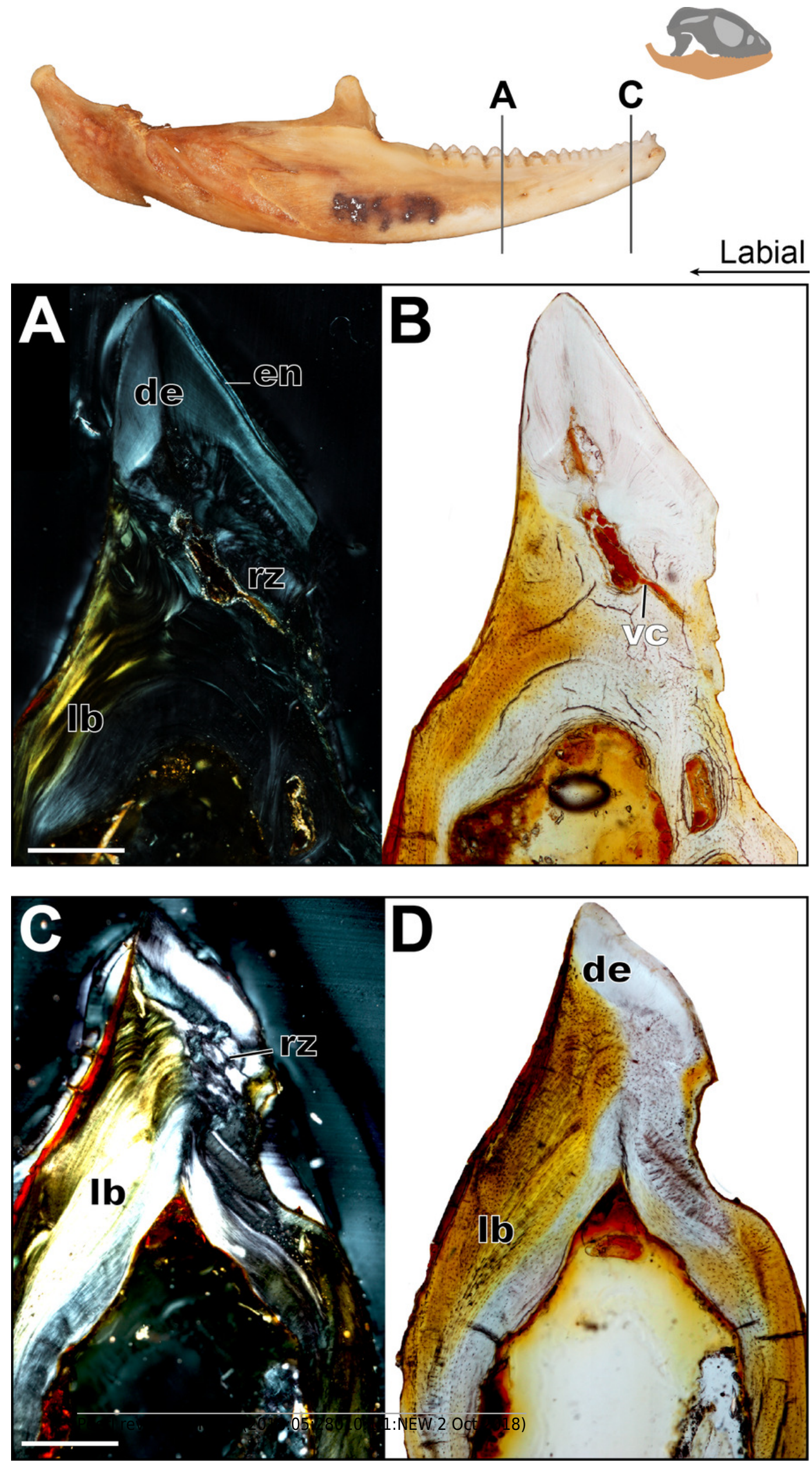


\section{Figure 7}

Schematic explanation of implantation changes through ontogeny and tooth wear.

(A) three basic traditional implantation categories, without attachment tissue illustrated; (B) pleurodont impanated tooth is attached via alveolar bone, yellow arrows show direction of dentine deposition; (C) purple arrows show direction of dentine resorption by odontoclasts, blue arrow shows direction of bone deposition by odontoblasts; (D) blue arrow shows further bone deposition, trabecula in the dentary is reduced; (E) the tooth appears implanted at the apex of the dentary; (F) Shows the position of maxillary tooth; (G) the enamel is worn on the labial side; $(\mathrm{H})$ with advanced wear, the enamel, dentine, and dentary bone are worn on the labial side. 

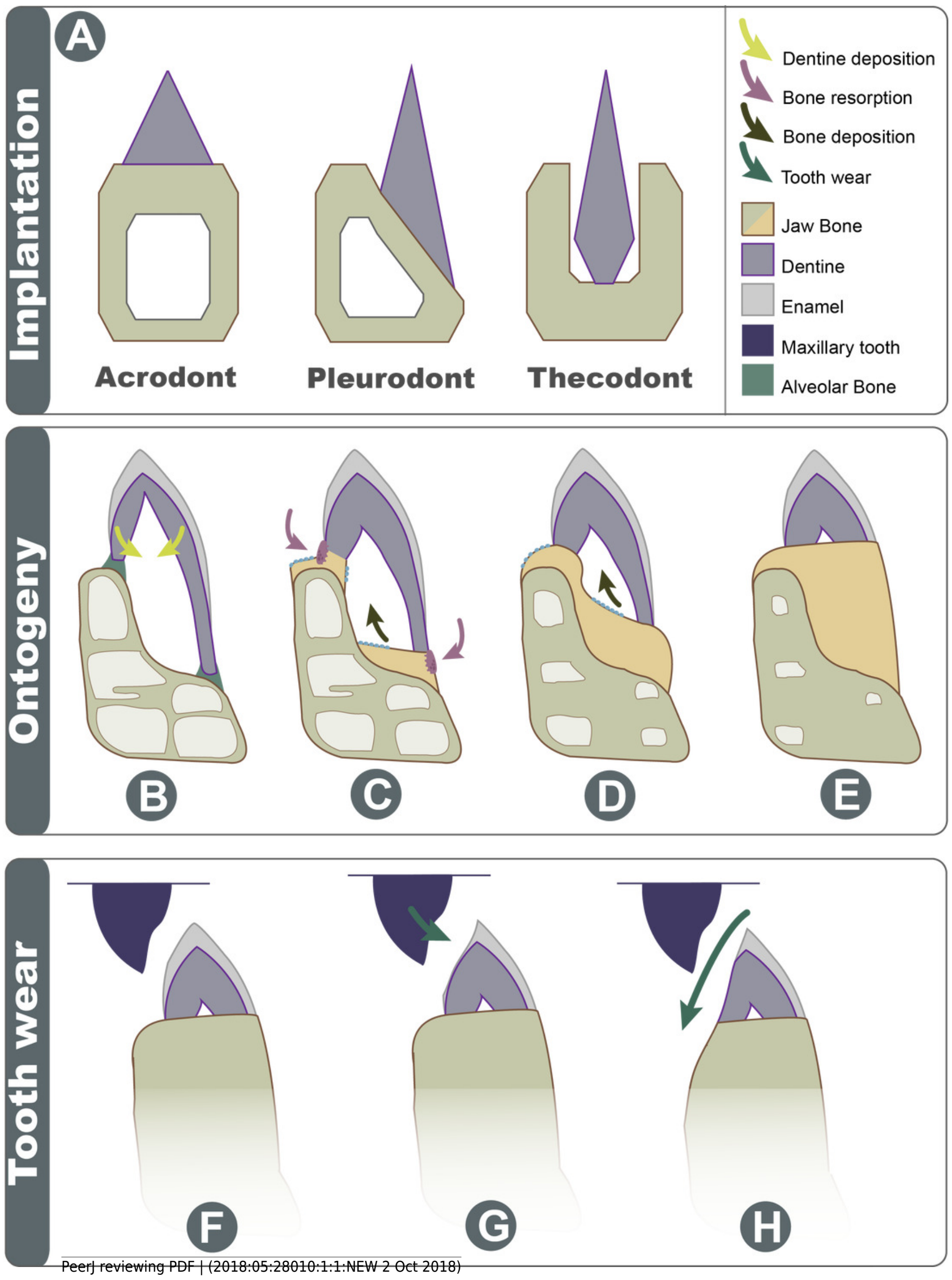